\title{
Can Achalasia Subtyping by High-Resolution Manometry Predict the Therapeutic Outcome of Pneumatic Balloon Dilatation?: Author's Reply
}

TO THE EDITOR: We would like to appreciate the interest shown in our article by Dr. Joo. Our response to his queries is as follows:

(1) The risk factors for prediction of recurrence after endoscopic pneumatic dilatation are young age, male sex, shorter duration of symptoms, low pre-treatment lower esophageal sphincter (LES) pressure and post therapy LES pressure above $10 \mathrm{mmHg}$. In the present study, the response to pneumatic dilatation was not influenced by sex and age. ${ }^{1}$ In a retrospective study of 52 patients by Mehta et $\mathrm{al}^{2}$ regarding the response of achalasia to pneumatic dilatation, there was no significant difference with respect to gender but younger age was associated with poor response in the Indian subpopulation. The reason why pneumatic dilatation is less effective in male is not known while Ghoshal et $\mathrm{al}^{3}$ have hypothesized that this might be due to the stronger LES musculature in men.

The previous studies have shown that younger patients had a poor outcome. The Indian sub-population studies by Ghoshal et $\mathrm{al}^{4}$ have shown that age did not affect the outcome of pneumatic dilatation which is similar to our study. The explanation for the worse outcome with young age is unknown.

Post pneumatic dilatation manometry was not done in the present study. In the present study, the statistically insignificant association between age and gender with the outcome may be due to the smaller number of patients in each of the subtypes. ${ }^{1}$

(2) In our study, the failure of therapy was defined as the persistence of symptoms for 4 weeks after pneumatic dilatation and need for further dilatations. The definition of successful treatment has been described in the methodology of the article.
(3) The major finding in our study was that the typing of achalasia predicted the response to pneumatic dilatation. We agree that the number of patients in our study was small. A study by Pandolfino et $\mathrm{al}^{5}$ has shown similar results. A larger prospective study with a large number of patients in each subtype and long-term follow-up is needed to further validate the findings of our study.

Nitesh Pratap and D Nageshwar Reddy
Asian Institute of Gastroenterology
Hyderabad, India

1. Pratap N, Kalapala R, Darisetty S, et al. Achalasia cardia subtyping by high-resolution manometry predicts the therapeutic outcome of pneumatic balloon dilatation. J Neurogastroenterol Motil 2011;17: 48-53.

2. Mehta R, John A, Sadasivan S, et al. Factors determining successful outcome following pneumatic balloon dilation in achalasia cardia. Indian J Gastroenterol 2005;24:243-245.

3. Ghoshal UC, Rangan M. A review of factors predicting outcome of pneumatic dilation in patients with achalasia cardia. J Neurogastroenterol Motil 2011;17:9-13.

4. Ghoshal UC, Kumar S, Saraswat VA, Aggarwal R, Misra A, Choudhuri G. Long-term follow-up after pneumatic dilation for achalasia cardia: factors associated with treatment failure and recurrence. Am J gastroenterol 2004;99:2304-2310.

5. Pandolfino JE, Kwiatek MA, Nealis T, Bulsiewicz W, Post J, Kahrilas PJ. Achalasia: a new clinically relevant classification by high-resolution manometry. Gastroenterology 2008;135:1526-1533.

Conflicts of interest: None. 